\title{
Frequency-radial duality based photoacoustic image reconstruction
}

\author{
S. M. Akramus Salehin ${ }^{\mathrm{a})}$ and Thushara D. Abhayapala \\ Applied Signal Processing Group, Research School of Engineering, College of Engineering and Computer Science, \\ Australian National University, Canberra, ACT 0200, Australia
}

(Received 21 July 2011; revised 7 February 2012; accepted 1 May 2012)

\begin{abstract}
Photoacoustic image reconstruction algorithms are usually slow due to the large sizes of data that are processed. This paper proposes a method for exact photoacoustic reconstruction for the spherical geometry in the limiting case of a continuous aperture and infinite measurement bandwidth that is faster than existing methods namely (1) backprojection method and (2) the Norton-Linzer method [S. J. Norton and M. Linzer, "Ultrasonic reflectivity imaging in three dimensions: Exact inverse scattering solution for plane, cylindrical and spherical apertures," Biomedical Engineering, IEEE Trans. BME 28, 202-220 (1981)]. The initial pressure distribution is expanded using a spherical Fourier Bessel series. The proposed method estimates the Fourier Bessel coefficients and subsequently recovers the pressure distribution. A concept of frequency-radial duality is introduced that separates the information from the different radial basis functions by using frequencies corresponding to the Bessel zeros. This approach provides a means to analyze the information obtained given a measurement bandwidth. Using order analysis and numerical experiments, the proposed method is shown to be faster than both the backprojection and the Norton-Linzer methods. Further, the reconstructed images using the proposed methodology were of similar quality to the Norton-Linzer method and were better than the approximate backprojection method.
\end{abstract}

(C) 2012 Acoustical Society of America. [http://dx.doi.org/10.1121/1.4725767]

PACS number(s): 43.35.Ud, 43.60.Pt, 43.60.Rw, 43.60.Lq [TDM]

Pages: $150-161$

\section{INTRODUCTION}

Photoacoustic image reconstruction is an inverse problem requiring the estimation of the initial spatial distribution of pressure by processing a set of recorded signals. However, existing reconstruction methods ${ }^{1-3}$ deal with a large amount of data due to the high measurement bandwidths. A novel frequency domain method is proposed for the spherical sensor configuration where the spatial pressure distribution is expanded using a spherical Fourier Bessel series. By processing only the frequencies corresponding to the Bessel zeros, the Fourier Bessel coefficients can be estimated and the spatial distribution recovered. This reduction in data processing provides a faster solution than previous reconstruction methods. Further, new insights relating the amount of information processing required with the spatial characteristics are provided.

Photoacoustic imaging has attracted considerable interest in recent years. This imaging method has the high resolution of ultrasound imaging and the high contrast of optical imaging. ${ }^{4}$ In photoacoustic imaging, light absorbing particles can be visualized, providing valuable information for medical diagnosis. Photoacoustic imaging is especially applicable in biomedical scenarios. These applications include cancer detection where tumors have a high light absorption coefficient, imaging blood vessels, imaging small animals and for imaging external organs.

\footnotetext{
a) Author to whom correspondence should be addressed. Also at National ICT Australia, Canberra, ACT 2601, Australia. Electronic address: asalehin@rsise.anu.edu.au.
}

This paper considers the case where the sensors are placed in a spherical configuration surrounding the sample under study and the spatial distribution is bounded within a known radius. Other configurations are possible and reconstruction algorithms have been provided for these different setups. ${ }^{3,5-9}$ The spherical configuration can be useful for imaging external organs, small animals or testing blood samples for cancer. A Fourier series ${ }^{1}$ and a time domain backprojection method ${ }^{2}$ was proposed for image reconstruction for the spherical geometry. Speed of image reconstruction is usually slow using these algorithms since a large number of samples need to be processed. More efficient and faster reconstruction algorithms are still needed for photoacoustic imaging.

The main contribution of this paper are as follows. A new method for photoacoustic image reconstruction is proposed which expands the spatial pressure distribution using a spherical Fourier Bessel series. A natural basis function (Fourier Bessel series) for the radial component is introduced which has not been previously mentioned in the photoacoustic literature. The Fourier Bessel series has been applied previously for solving boundary value problems ${ }^{10}$ and for scattering in nuclear or atomic systems. ${ }^{11,12}$ The method proposed in this paper can be applied for inverse problems involving a wave equation and a frequency invariant compact source. The proposed method processes only the frequencies corresponding to the Bessel zeros to estimate the spherical Fourier coefficients and subsequently, recover the spatial distribution. A new concept of frequency-radial duality is introduced which shows that sampling at the Bessel zero frequencies separates the information from the different radial basis functions. The 
proposed method provides exact reconstruction only in the continuous limit where sampling is ignored. Further, a discretization of this exact method for practical implementation is proposed.

Frequency-radial duality provides new insights into the information content given a finite bandwidth and volume of space occupied by the initial pressure distribution. The proposed method is proved to be faster than both the backprojection $^{2}$ and the Fourier series (Norton-Linzer) ${ }^{1}$ methods using order analysis and numerical experiments. Furthermore, the computational order analysis provides conditions under which the Norton-Linzer method is faster than the backprojection algorithm. Moreover, spatial aliasing ideas from spherical array signal processing are extended for photoacoustic imaging.

This paper is organized as follows. The next section provides some theoretical background to the underlying wave equation associated with photoacoustic imaging. Section III outlines the problem statement, provides a description of the spherical Fourier Bessel expansion of the initial spatial distribution of pressure and introduces the proposed frequency-radial duality (F-R) based algorithm. Section IV extends the proposed method considering aliasing and discrete spatial sampling. Section V compares the F-R based method with the Norton-Linzer and the backprojection methods. Further, a computational order analysis is provided for these three methods. A description of the numerical experiments performed to validate and compare the F-R based method with previous methods are provided in Sec. VI. Section VII concludes the paper by providing a summary of the main ideas.

\section{BACKGROUND ON PHOTOACOUSTIC IMAGING}

This section provides a brief overview of photoacoustic imaging together with the solutions to the wave equations associated with it. Photoacoustic imaging uses pressure waves generated by the absorption of a laser pulse. It is generally assumed that the incident electromagnetic (EM) wave is absorbed instantaneously within the targeted volume of tissue. This condition can be met when the incident laser pulse duration is short such that the temporal illumination function can be approximated by a Dirac delta function $\delta(t)$. Assuming constant speed of propagation $c$ in the medium, the pressure $p(\boldsymbol{r}, t)$ at a vector position $\boldsymbol{r}$ and time $t$ in a lossless, linear medium is described by the inhomogeneous Helmholtz's equation ${ }^{13,14}$

$$
\frac{\partial^{2} p(\boldsymbol{r}, t)}{\partial t^{2}}-c^{2} \cdot \nabla^{2} p(\boldsymbol{r}, t)=p_{0}(\boldsymbol{r}) \frac{\partial}{\partial t} \delta(t),
$$

where the time derivative of the impulsive pressure distribution $p_{0}(\boldsymbol{r}) \delta(t)$ provides the driving force. The initial, spatial distribution of pressure is denoted by $p_{0}(\boldsymbol{r})$, however, this will be simply referred to as the spatial distribution in the rest of this paper.

The spatial distribution is linearly related to the distribution of the EM absorption coefficient $\mu(\boldsymbol{r})$ by

$$
p_{0}(\boldsymbol{r})=\Gamma \Psi(\boldsymbol{r}) \mu(\boldsymbol{r}),
$$

where $\Psi(\boldsymbol{r})$ is the optical fluence distribution and $\Gamma$ is the dimensionless Grüneisen coefficient which is defined by $\Gamma=\varrho c^{2} / C_{p}$ with $\varrho$ the volume expansion sensitivity and $C_{p}$ the isobaric specific heat capacity. By solving for $p_{0}(\boldsymbol{r})$, differences in the EM absorption property can be observed in the target volume. This can identify different types of tissues or cancerous regions which have different EM absorption coefficients.

The pressure recorded by an ultrasound transducer in the time domain and at a position $\boldsymbol{r}_{s}$ can be described by the general solution to the inhomogeneous Helmholtz equation ${ }^{10}$ (Chap. 9) (1), which is expressed as

$$
p\left(\boldsymbol{r}_{s}, t\right)=\frac{\partial}{\partial t} \int_{V} p_{0}(\boldsymbol{r}) \frac{\delta\left(t-\left\|\boldsymbol{r}_{s}-\boldsymbol{r}\right\| / c\right)}{4 \pi\left\|\boldsymbol{r}_{s}-\boldsymbol{r}\right\|} d \boldsymbol{r}
$$

where $\int_{V} \mathrm{~d} \boldsymbol{r}$ represents integration over a volume of space in $\mathbb{R}^{3}$ and $\|\cdot\|$ is a vector norm. The time domain solution is used in several reconstruction algorithms. ${ }^{5,6}$ Moreover, the Fourier transform of the measured acoustic wave (3) over time $t$ can be written as

$$
p\left(\boldsymbol{r}_{s}, k\right)=-i k c \int_{V} p_{0}(\boldsymbol{r}) G\left(k ; \boldsymbol{r}_{s}, \boldsymbol{r}\right) d \boldsymbol{r},
$$

where $i \triangleq \sqrt{-1}$, the wavenumber $k=2 \pi f / c, f$ denotes the frequency and $G(\cdot)$ is the Green's function. This Fourier transform was used in photoacoustic image reconstruction methods described in Refs. 3 and 7. From (4), the spatial distribution $p_{0}(\boldsymbol{r})$ is frequency invariant, hence the estimation of such a distribution is classified as a frequency invariant, distributed source localization problem.

\section{A. Wavefield decomposition}

In the previous section the Green's function was mentioned and this function in $\mathbb{R}^{3}$ using the standard spherical co-ordinate system $^{10}$ (Chap. 9) is defined as

$$
G\left(k ; \boldsymbol{r}_{s}, \boldsymbol{r}\right) \triangleq \frac{e^{i k\left\|\boldsymbol{r}_{s}-\boldsymbol{r}\right\|}}{4 \pi\left\|\boldsymbol{r}_{s}-\boldsymbol{r}\right\|}
$$

with sensor position $\boldsymbol{r}_{s} \triangleq\left(r_{s}, \Omega_{s}\right)$, source position $\boldsymbol{r} \triangleq(r, \Omega)$, $\Omega_{s} \triangleq\left(\theta_{s}, \phi_{s}\right)$ and $\Omega \triangleq(\theta, \phi)$. Next, we introduce the wavefield or modal decomposition of the Green's function as a sum of orthogonal basis functions ${ }^{15}$ (p. 259),

$$
G\left(k ; \boldsymbol{r}_{s}, \boldsymbol{r}\right)=\sum_{n, m} \boldsymbol{r}_{n}\left(k ; r_{s}, r\right) Y_{n m}\left(\Omega_{s}\right) Y_{n m}^{*}(\Omega),
$$

where

$$
R_{n}\left(k ; r_{s}, r\right)=i k h_{n}^{(1)}\left(k r_{s}\right) j_{n}(k r)
$$

valid for $k>0$ and $r_{s}>r$; with $\sum_{n, m} \triangleq \sum_{n=0}^{\infty} \sum_{m=-n}^{n}, n$ as the order, $m$ as the mode, $h_{n}^{(1)}(\cdot)$ and $j_{n}(\cdot)$ are the spherical Hankel function of the first kind and the spherical Bessel function, respectively, and ${ }^{*}$ denotes the complex conjugate operator. The spherical harmonics are denoted by $Y_{n m}(\cdot)$ and are defined as 


$$
Y_{n m}(\Omega) \triangleq \sqrt{\frac{(2 n+1)}{4 \pi} \frac{(n-m) !}{(n+m) !}} P_{n m}(\cos \theta) e^{i m \phi},
$$

where the polynomial function $P_{n m}(\cdot)$ is the associated Legendre function.

This decomposition introduced here will be used for deriving our proposed algorithm and was used previously in ultrasound reflectivity imaging. ${ }^{1}$ This modal decomposition has the advantage of separating components dependent on the source position form those dependent on the sensor position and thus found application in direction of arrival (DOA) estimation ${ }^{16}$ and in biomedical acoustic source localization within circular sensor arrays. ${ }^{17,18}$

\section{FREQUENCY-RADIAL DUALITY BASED IMAGE RECONSTRUCTION}

This section introduces our novel algorithm for photoacoustic imaging under certain ideal conditions. Extension for practical application is considered in the subsequent section. In this section we elaborate the problem statement, the novel spherical Fourier Bessel expansion of the spatial distribution, and highlight the two components of our proposed method for photoacoustic tomography.

\section{A. Problem statement}

We develop our algorithm by considering a hypothetical continuous aperture on the 2-sphere $\mathbb{S}^{2}$ at a radius of $r_{s}$. Further, the problem of photoacoustic image reconstruction is an inverse problem requiring the estimation of the spatial distribution $p_{0}(r, \Omega)$ [specifying $p_{0}(\boldsymbol{r})$ in expression (4) in a standard spherical co-ordinate system] from measurements $p\left(\Omega_{s}, k\right)$ (specifying the measured pressure $p\left(\boldsymbol{r}_{s}, k\right)$ due to acoustic waves on a spherical manifold at $r_{s}$ ). This paper considers the case where the sensor aperture is specified by samples on the sphere $\mathbb{S}^{2}$ completely enclosing the spatial distribution and Fig. 1 illustrates the geometry of the problem formulation, with the spatial distribution being zero at all spatial points with radii larger than $r_{0}$.

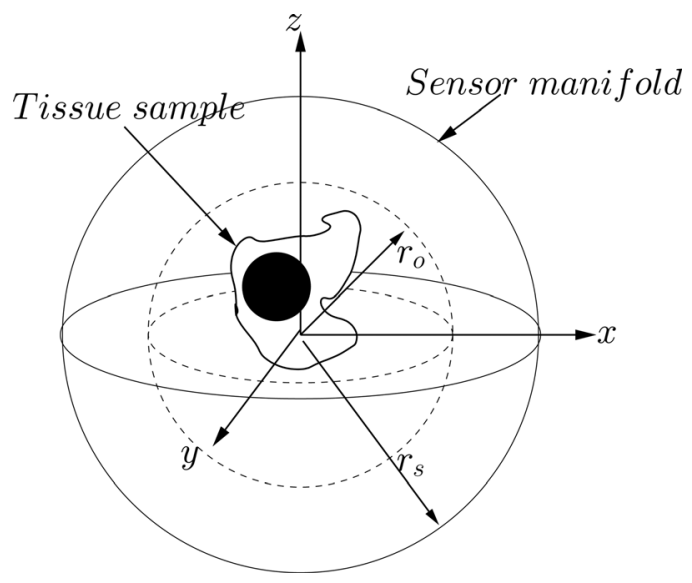

FIG. 1. The problem involves estimating the spatial distribution enclosed in a spherical region of radius $\boldsymbol{r}_{0}$ from measurements on a continuous spherical aperture at a radius $\boldsymbol{r}_{s}$, completely enclosing the bounding region.

\section{B. Fourier transform on the 2-sphere}

The spherical Harmonic functions form a complete orthonormal basis function on the 2 -sphere $\mathbb{S}^{2}$ with respect to the natural inner product. ${ }^{15}$ Provided that the aperture response is square integrable on the sphere, i.e., $p(\cdot, k)$ $\in \mathcal{L}^{2}\left(\mathbb{S}^{2}\right)$, the spherical harmonics decomposition ${ }^{15}$ (Chap. 6 ), or the spherical Fourier transform ${ }^{19}$ analysis and synthesis equations of the aperture response function at wavenumber $k$ is, respectively,

$$
\wp_{n m}(k)=\int_{\Omega_{s} \in \mathbb{S}^{2}} p\left(\Omega_{s}, k\right) Y_{n m}^{*}\left(\Omega_{s}\right) d \Omega_{s}
$$

and

$$
p\left(\Omega_{s}, k\right)=\sum_{n, m} \wp_{n m}(k) Y_{n m}\left(\boldsymbol{\Omega}_{s}\right),
$$

where $\int_{\Omega_{s} \in \mathbb{S}^{2}} d \Omega_{s} \triangleq \int_{0}^{2 \pi} \int_{0}^{\pi} \sin \theta_{s} d \theta_{s} d \phi_{s}$ and represents integration over the unit sphere. The spherical Fourier coefficients $\wp_{n m}$ are complex terms and equality in expression (10) means convergence in the mean, however, if the aperture function is sufficiently smooth, point-wise convergence can be assumed. The spherical Fourier transform introduced in this section will be applied in later parts of this paper.

\section{Spherical fourier bessel expansion of spatial distribution}

The spherical Fourier Bessel series ${ }^{20,21}$ forms an orthonormal basis on an interval $\left[0, r_{0}\right)$. A function $f(r)$ defined on this interval can be expanded with the $n^{\text {th }}$ order, spherical Fourier Bessel series as $f(r)=\sum_{\ell=1}^{\infty} \widehat{\alpha}_{\ell} j_{n}\left(\frac{z_{n \ell}}{r_{0}} r\right)$ where $z_{n \ell}$ is the $\ell$ th root of $j_{n}(\cdot), \ell$ denotes the Bessel zero index and $\hat{\alpha}_{\ell}$ are Bessel coefficients. This series is applied for boundary value problems, ${ }^{10}$ for simulation of cosmic microwave backgrounds $^{22}$ and for atomic scattering problems. ${ }^{11,12}$

The spatial distribution $p_{0}(\boldsymbol{r})$ can be expanded as a sum of orthogonal basis functions (synthesis equation)

$$
p_{0}(\boldsymbol{r})=\sum_{n, m} \sum_{\ell=1}^{\infty} \alpha_{n m \ell} j_{n}\left(\frac{z_{n \ell}}{r_{0}} r\right) Y_{n m}(\Omega)
$$

where $\alpha_{n m \ell}$ are complex, spherical Fourier Bessel coefficients. Note that $\ell$ also acts as an index for the radial basis function and will also be called the radial index. In this expansion, the angular components are expanded with the spherical harmonic functions and the radial component is expanded with the spherical Fourier Bessel series. Similar expansions were used in Refs. 20 and 21.

This expansion will be referred to as the spherical Fourier Bessel expansion. The corresponding analysis equation to calculate $\alpha_{n m \ell}$ can be written as

$$
\alpha_{n m \ell}=\frac{2}{r_{0}^{3} j_{n+1}^{2}\left(z_{n \ell}\right)} \int_{0}^{r_{0}} \int_{\Omega \in \mathbb{S}^{2}} p_{0}(\boldsymbol{r}) Y_{n m}^{*}(\Omega) j_{n}\left(\frac{z_{n \ell}}{r_{0}} r\right) d \Omega r^{2} d r .
$$

The analysis equation can be explained by the spherical Fourier transform described in Sec. III B and by the spherical 
Fourier Bessel series, which is used for the radial basis function. This spherical Fourier Bessel series is derived from the well known Fourier Bessel series which found application in expanding acoustic wavefields. ${ }^{20}$

If we can estimate all the spherical Fourier Bessel coefficients $\alpha_{n m \ell}$ then we can reconstruct the spatial distribution $p_{0}(\boldsymbol{r})$ using the synthesis equation (11). Such an orthogonal expansion (11) has not been proposed before in either photoacoustic or ultrasonic reflectivity imaging. However, the authors have previously introduced a 2D Fourier Bessel expansion for the spatial distribution which was applied for $2 \mathrm{D}$ photoacoustic imaging in a circular geometry ${ }^{23}$ and for 2.5D photoacoustic reconstruction for a cylindrical geometry. $^{24}$

\section{Modal-order filtering of the spatial distribution}

So far we have introduced the wave equations related to photoacoustic imaging, the wavefield modal expansion and the spherical Fourier Bessel expansion. This section describes a methodology that can separate the information from the different modes and orders in the spatial distribution expansion (11).

Firstly, we define a new function

$$
\beta_{n m}(\boldsymbol{r})=\sum_{\ell=1}^{\infty} \alpha_{n m \ell} j_{n}\left(\frac{z_{n \ell}}{r_{0}} r\right)
$$

thus, the spatial distribution expansion can be shortened to

$$
p_{0}(\boldsymbol{r})=\sum_{n, m} \beta_{n m}(r) Y_{n m}(\Omega) .
$$

This definition allows a simpler notation in describing the following Theorem.

Theorem 1 (Modal-Order Filtering). Taking the spherical Fourier transform of the aperture response $p\left(\Omega_{s}, k\right)$ separates the information from the different orders $n$ and modes $m$ in the spherical Fourier Bessel expansion of the spatial distribution. Hence, the spherical Fourier coefficient $\wp_{n, m}(k)$ of order $n$ and mode $m$ is only dependent on the coefficients of the same order and mode in the spherical Fourier Bessel expansion of the spatial distribution $p_{0}(\boldsymbol{r})$, i.e.,

$$
\wp_{n m}(k)=-i k c \int_{0}^{r_{0}} \beta_{n m}(r) R_{n}\left(k ; r_{s}, r\right) r^{2} d r .
$$

Proof. By substituting the Fourier Bessel expansion (14) and the modal expansion of the Green's function (6) into (4), the received signal by a sensor at angular position $\Omega_{s}$ for wavenumber $k$ is

$$
\begin{aligned}
p\left(\Omega_{s}, k\right)= & -i k c \int_{V} \sum_{n^{\prime} m^{\prime}} \beta_{n^{\prime}, m^{\prime}}(r) Y_{n^{\prime} m^{\prime}}(\Omega) \\
& \times \sum_{n, m} R_{n}\left(k ; r_{s}, r\right) Y_{n m}^{*}(\Omega) Y_{n m}\left(\Omega_{s}\right) d V \\
= & -i k c \sum_{n^{\prime}, m^{\prime}} \sum_{n, m} \int_{\Omega \in \mathbb{S}^{2}} Y_{n^{\prime} m^{\prime}}(\Omega) Y_{n m}^{*}(\Omega) d \Omega \\
& \times \int_{0}^{r_{0}} \beta_{n^{\prime} m^{\prime}}(r) R_{n}\left(k ; r_{s}, r\right) r^{2} d r Y_{n m}\left(\Omega_{s}\right) .
\end{aligned}
$$

This can be simplified further by applying the orthogonality property of the spherical harmonic functions in the first integral, over the angular domain to yield

$$
\begin{aligned}
& p\left(\Omega_{s}, k\right) \\
& =\sum_{n, m} \underbrace{\left(-i k c \int_{0}^{r_{0}} \beta_{n m}(r) R_{n}\left(k ; r_{s}, r\right) r^{2} d r\right)}_{\wp_{n m}(k)} Y_{n m}\left(\Omega_{s}\right) .
\end{aligned}
$$

By comparing (17) to (10), the spherical Fourier transform of the aperture response for order $n$ and mode $m$ is (15).

The advantage of choosing separable basis expansions for the radial and angular components in both the wavefield modal expansion and the spatial distribution expansion allows the integration over a spherical volume $V$ to be separated to two independent integrals (16). One of these integrals is over the radial parameter while the other is over the angular parameter. Further, the separation of integrals allows the exploitation of the orthogonality property of the spherical harmonic functions reducing summation over four parameters to summation over two (17).

So far, we can separate out the information from the different orders and modes, however, to estimate the spherical Fourier Bessel coefficients $\alpha_{n m \ell}$ in (13) a further separation of the radial indices is required.

\section{E. Frequency-radial duality}

The following theorem describes a method to separate the information from the different radial basis functions

Theorem 2 (Frequency-Radial Duality). The spherical Fourier coefficient $\wp_{n m}(k)$ taken at frequencies corresponding to the Bessel zeros, more specifically at $k=z_{n \ell} / r_{0}$, is dependent only on the spherical Fourier Bessel coefficient corresponding to the Bessel zero index $\ell$ and given by

$$
\left.\wp_{n m}(k)\right|_{k=z_{n \ell} / r_{0}}=\frac{\left(z_{n \ell}\right)^{2}}{2} c r_{0} j_{n+1}^{2}\left(z_{n \ell}\right) h_{n}^{(1)}\left(\frac{z_{n \ell}}{r_{0}} r_{s}\right) \alpha_{n m \ell} .
$$

Proof. Substituting (7) and (13) into (15), we get the spherical Fourier coefficient

$$
\begin{aligned}
\wp_{n m}(k)= & k^{2} c \sum_{\ell^{\prime}=1}^{\infty} h_{n}^{(1)}\left(k r_{s}\right) \\
& \times \int_{0}^{r_{0}} \alpha_{n m \ell^{\prime}} j_{n}\left(\frac{z_{n \ell^{\prime}}}{r_{0}} r\right) j_{n}(k r) r^{2} \mathrm{~d} r
\end{aligned}
$$

and for $k=z_{n \ell} / r_{0}$ this becomes

$$
\begin{aligned}
\wp_{n m}\left(\frac{z_{n \ell}}{r_{0}}\right)= & \left(\frac{z_{n \ell}}{r_{0}}\right)^{2} c \sum_{\ell^{\prime}=1}^{\infty} \alpha_{n m \ell^{\prime}} h_{n}^{(1)}\left(\frac{z_{n \ell}}{r_{0}} r_{s}\right) \\
& \times \int_{0}^{r_{0}} j_{n}\left(\frac{z_{n \ell^{\prime}}}{r_{0}} r\right) j_{n}\left(\frac{z_{n \ell}}{r_{0}} r\right) r^{2} \mathrm{~d} r .
\end{aligned}
$$

The integral in (20) can be simplified by using the orthogonality property of the spherical Bessel function ${ }^{25}$ 


$$
\begin{aligned}
& \int_{0}^{r_{0}} j_{n}\left(\frac{z_{n \ell}}{r_{0}} r\right) j_{n}\left(\frac{z_{n \ell^{\prime}}}{r_{0}} r\right) r^{2} d r \\
& \quad= \begin{cases}\frac{r_{0}^{3}}{2} j_{n+1}^{2}\left(z_{n \ell}\right) & \text { if } \ell=\ell^{\prime} \\
0 & \text { otherwise. }\end{cases}
\end{aligned}
$$

Now, the spherical Fourier coefficient $\wp_{n m}\left(z_{n \ell} / r_{0}\right)$ in (20) can be written as

$$
\wp_{n m}\left(\frac{z_{n \ell}}{r_{0}}\right)=\left(\frac{z_{n \ell}}{r_{0}}\right)^{2} c \frac{r_{0}^{3} j_{n+1}^{2}\left(z_{n \ell}\right)}{2} h_{n}^{(1)}\left(\frac{z_{n \ell}}{r_{0}} r_{s}\right) \alpha_{n m \ell}
$$

and with further algebraic simplification yields (18).

From the aperture response $p\left(\Omega_{s}, k\right)$, we can get $\wp_{n m}\left(z_{n \ell} / r_{0}\right)$ by applying the spherical Fourier transform at $k=z_{n \ell} / r_{0}$. Subsequently, using the result obtained in (18), we can estimate the spherical Fourier Bessel coefficient $\alpha_{n m \ell}$ of the spatial distribution $p_{0}(\boldsymbol{r})$ by

$$
\alpha_{n m \ell}=\frac{2 \wp_{n m}\left(z_{n \ell} / r_{0}\right)}{\left(z_{n \ell}\right)^{2} c r_{0} j_{n+1}^{2}\left(z_{n \ell}\right) h_{n}^{(1)}\left(\left[z_{n \ell} / r_{0}\right] r_{s}\right)} .
$$

Measurements are available over different angular positions, however, the different frequency samples provides the extra dimension required to derive the radial information of the spatial distribution $p_{0}(\boldsymbol{r})$. This is shown by Theorem 2 .

In practice the sensor has a finite frequency response, let $k_{l}$ denote the lower limit and $k_{u}$ the upper limit of this frequency response. The coefficients $\alpha_{n m \ell}$ that can be estimated must have radial indices $\ell$ and order $n$ such that $k_{l} \leq z_{n \ell} / r_{0}$ $\leq k_{u}$. Therefore, only a finite number of coefficients $\alpha_{n m \ell}$ can be obtained due to the finite bandwidth of measurement and so an estimate of $p_{0}(\boldsymbol{r})$ is reconstructed. The number of orders $n$ that needs to be accounted depends only on the upper frequency limit $k_{u}$. This is due to the fact that the value of the first root $z_{n, 1}$ increases with order $n$ for the spherical Bessel functions. Thus, the largest order $n$ satisfies

maximize $n$

such that $\frac{z_{n, 1}}{r_{0}} \leq k_{u}$.

It is intuitive that to estimate $p_{0}(\boldsymbol{r})$ which occupies a larger area of space would require more information or more samples. This idea is proved by our proposed method where $r_{0}$ specifies a bounding radius and affects the location of frequency samples. If $r_{0}$ is large then the spacing between adjacent frequency samples used for the same order decreases, that is,

$$
k_{i+1}-k_{i}=\frac{z_{n, i+1}}{r_{0}}-\frac{z_{n, i}}{r_{0}}=\frac{z_{n, i+1}-z_{n, i}}{r_{0}} .
$$

Therefore, more frequency samples are used for a given measurement bandwidth and that a larger number of coefficients $\alpha_{n m \ell}$ can be obtained from the same measurement bandwidth. The effect of the bounding radius on the information required has not been previously mentioned in the photoacoustic literature and cannot be accounted for by the previous proposed algorithms. Our method shows that the smaller the area occupied by $p_{0}(\boldsymbol{r})$ within the sensor manifold, the less the information that is required for reconstruction (lower number of frequency samples need to be processed) and so the reconstruction is faster.

\section{F. Algorithm simplification}

To estimate $p_{0}(\boldsymbol{r})$, we only need to estimate half the spherical Fourier Bessel coefficients $\alpha_{n m \ell}$ due to the fact that the spatial $p_{0}(\boldsymbol{r})$ is a real function. Therefore, $p_{0}(\boldsymbol{r})=p_{0}^{*}(\boldsymbol{r})$, and so the complex conjugate of the spherical Fourier Bessel expansion is

$$
p_{0}^{*}(\boldsymbol{r})=\sum_{n, m} \sum_{\ell=1}^{\infty} \alpha_{n m \ell}^{*} j_{n}\left(\frac{z_{n \ell}}{r_{0}} r\right) Y_{n m}^{*}(\Omega) .
$$

Note that, unlike the other terms, the spherical Bessel functions are always real. Further, if we exploit the following relationship $Y_{n m}^{*}(\Omega)=(-1)^{m} Y_{n(-m)}(\Omega)^{15}$ then

$$
p_{0}(\boldsymbol{r})=\sum_{n=0}^{\infty} \sum_{\ell=1}^{\infty} j_{n}\left(\frac{z_{n \ell}}{r_{0}} r\right) \sum_{m=-n}^{n} \alpha_{n m \ell}^{*}(-1)^{m} Y_{n(-m)}(\Omega) .
$$

From the previous equation, we can infer that

$$
\alpha_{n(-m) \ell}=(-1)^{m} \alpha_{n m \ell}^{*}
$$

and so if we can estimate $\alpha_{n m \ell}$, we can also obtain $\alpha_{n(-m) \ell}$. In practice we can choose to estimate $\alpha_{n m \ell}$ for only the positive modes $(m \geq 0)$.

\section{EXTENSION TO DISCRETE SPATIAL AND TEMPORAL SAMPLES}

In this section, we discuss the impact of discrete time and discrete spatial samples. For the case of discrete time samples, the sampling frequency must be more than twice the upper limit of the frequency response of the sensor. This condition ensures that "temporal aliasing" is avoided.

In this paper, we do not consider the blurring effect caused by the finite size of the sensor but assume that sensors sample a single point in the spatial domain. In planar array geometries, spatial aliasing can be avoided by ensuring that the distance between the sensors is smaller than half the wavelength associated with the upper frequency limit of the sensor response. If the spherical Fourier transform of the aperture response is order limited, i.e., $\wp_{n m}(k)=0$ for $n>N$, to avoid spatial aliasing using an equiangular sampling method ${ }^{19}$ both azimuth $\phi$ and elevation $\theta$ are each sampled by $2 N+1$ equiangular spaced samples [total of $4(N+1)^{2}$ samples]. A method based on Gaussian quadrature only requires $N+1$ samples for the elevation. ${ }^{10}$ It is possible to space the sensors uniformly on the sphere, reducing the total number of sensors to $(N+1)^{2}$, however, there is no analytical expression for the sensor positions. $^{26}$

The order limiting value $N$ can be determined by borrowing a concept from spherical array signal processing. 
This concept states that aliasing is negligible provided that the product of the upper wavenumber limit $k_{u}$ of the sensor response and the radius of the sensors placement $r_{s}$ is smaller than $N,{ }^{16,27}$

$$
k_{u} r_{s}<N \text {. }
$$

In our case, we can assume that the order limit is provided from the spatial distribution expansion $p_{0}(\boldsymbol{r})$. Therefore, we assume that the Fourier Bessel coefficients $\alpha_{n m \ell}$ is negligible for $n>N$. For the rest of the paper, we employ this assumption and will only consider equiangular sensor placement.

The sphere is sampled using an equiangular sampling scheme where the discrete, inverse spherical Fourier transform is ${ }^{19}$

$$
\begin{aligned}
\wp_{n m}(k)= & \sum_{u=1}^{(2 N+1)} \sum_{q=1}^{(2 N+1)} a_{u q}\left(\theta_{q}\right) p\left(\Omega_{s, u q}, k\right) \\
& \times Y_{n m}^{*}\left(\Omega_{s, u q}\right) \sin \left(\theta_{q}\right),
\end{aligned}
$$

where the azimuth positions are indexed by $u$, the elevation by $q$ and the spatial weighting is ${ }^{19}$

$$
a_{u q}\left(\theta_{q}\right)=\frac{2 \pi}{N^{2}} \sum_{\kappa=0}^{N-1} \frac{\sin \left((2 \kappa+1) \theta_{q}\right)}{2 \kappa+1} .
$$

Other discrete spherical Fourier transforms use the Gaussian sampling scheme or the nearly uniform sampling scheme. A description of these two methods of computing the discrete spherical Fourier transform are provided in Ref. 28.

The proposed method does not require an inverse Fourier Bessel transform and only uses the synthesis part of the Fourier Bessel transform. Therefore, dicretization in the radial component is not utilized in the proposed method.

Another important point to consider is that if we use the fast Fourier transform (FFT) on the time domain data, then the frequency samples are uniformly spaced. The required frequency values corresponding to the Bessel zeros $k=z_{n \ell} / r_{0}$ may not be available. Provided that with zero padding, there are sufficient frequency samples, we can apply a simple linear interpolation method to calculate the frequency spectra corresponding to the Bessel zeros,

$$
\begin{aligned}
f\left(\Omega_{s}, \frac{z_{n \ell}}{r_{0}}\right)= & f\left(\Omega_{s}, k_{u}\right) \\
& +\frac{z_{n \ell} / r_{0}-k_{u}}{k_{u+1}-k_{u}}\left(f\left(\Omega_{s}, k_{u+1}\right)-f\left(\Omega_{s}, k_{u}\right)\right),
\end{aligned}
$$

where $f\left(\Omega_{s}, k\right)$ denotes the value of the frequency sample for sensor position $\Omega_{s}$ and at frequency $k, k_{u}$ is the frequency available directly smaller than $k=z_{n \ell} / r_{0}$ $\left(k=z_{n \ell} / r_{0}\right.$ lies between $k_{u}$ and $\left.k_{u+1}\right)$. The non-uniform Fourier transform can also solve this problem without using any interpolation. Moreover, for a better estimate other interpolation methods such as polynomial interpolation can be applied. However, this may increase the computational complexity.

\section{COMPARISON WITH PREVIOUS RECONSTRUCTION METHODS}

We compare our proposed method of photoacoustic tomography with another frequency domain, Fourier series method proposed by Norton and Linzer $^{1}$ for ultrasound reflectivity imaging which was later applied to photoacoustic imaging. ${ }^{2}$ We refer to this Fourier series method as the "Norton-Linzer" method. Subsequently, we evaluate theoretically, the computational complexity of our proposed method with two other photoacoustic imaging methods applicable to spherical geometries.

\section{A. Frequency domain algorithm}

The Norton-Linzer method ${ }^{1}$ for photoacoustic image reconstruction using the spherical geometry also specifies a bounding radius $r_{0}$ for the spatial distribution. This solution is briefly described in this subsection. This method utilizes modal-order filtering without specifying a basis expansion for the radial parameter. Taking the spherical Fourier transform over the aperture results $p\left(\Omega_{s}, k\right)$ in (17) yields

$$
\wp_{n m}(k)=k^{2} c \int_{0}^{r_{0}} \beta_{n m}(r) j_{n}(k r) r^{2} \mathrm{~d} r h_{n}^{(1)}\left(k r_{s}\right) .
$$

This series method utilizes a different orthogonality property of the spherical Bessel functions and over the wavenumber rather than the radius,

$$
\int_{0}^{\infty} k^{2} j_{n}(k r) j_{n}\left(k r^{\prime}\right) \mathrm{d} k=\frac{\pi}{2 r^{2}} \delta\left(r-r^{\prime}\right) .
$$

This orthogonality property is utilized to calculate $\beta_{n m}(r)$ for all $r$ by integrating the spherical Fourier coefficients multiplied a factor over all the frequency samples. This operation is formally described by

$$
\begin{aligned}
\int_{0}^{\infty} & \frac{\wp_{n m}(k)}{c h_{n}^{(1)}\left(k r_{s}\right)} j_{n}\left(k r^{\prime}\right) d k \\
\quad= & \int_{0}^{r_{0}} \beta_{n m}(r) \int_{0}^{\infty} k^{2} j_{n}(k r) j_{n}\left(k r^{\prime}\right) d k r^{2} d r \\
\quad= & \int_{0}^{r_{0}} \beta_{n m}(r) \frac{\pi}{2 r^{2}} \delta\left(r-r^{\prime}\right) r^{2} d r \\
= & \frac{\pi}{2} \beta_{n m}\left(r^{\prime}\right),
\end{aligned}
$$

by changing the subject of the resulting equation, the radial dependent coefficients are

$$
\beta_{n m}\left(r^{\prime}\right)=\frac{2}{\pi} \int_{0}^{\infty} \frac{\wp_{n m}(k)}{c h_{n}^{(1)}\left(k r_{s}\right)} j_{n}\left(k r^{\prime}\right) d k
$$

Subsequently, the spatial distribution can be reconstructed by evaluating the following equation for all reconstruction points 


$$
p_{0}(\boldsymbol{r})=\sum_{n, m} \beta_{n m}(r) Y_{n m}(\Omega) .
$$

The proposed reconstruction methodology and the NortonLinzer solution produce exact reconstruction if the measurement bandwidth in infinite and the spherical aperture is continuous, i.e., ignoring sampling effects. However, ultrasound transducers used in photoacoustic imaging have a finite measurement bandwidth, hence, the orthogonality property of the spherical Bessel functions in (34) utilized in the Norton-Linzer solution may not be valid. Further, the Norton-Linzer solution requires an extra operation of integrating over all frequency samples which is avoided in our methodology by sampling at frequencies corresponding to the Bessel zeros and allowing the propagation channel and the spherical configuration to perform a natural integration over the radius. Utilizing integration over the radius has the advantage of avoiding errors due to discretization of a continuous integral. Furthermore, the integration over the frequency increases the computation complexity of the series solution and all spherical Fourier coefficients needs to be calculated at all frequencies. Our methodology is more efficient requiring the spherical Fourier coefficients for a single order and only for frequencies corresponding to the Bessel zeros.

The direct calculation of the spherical Fourier Bessel coefficients can provide an efficient means of storage of the image if it is sparse in this domain rather than directly storing the samples at discrete points of the spatial distribution. Further, extra calculation to convert to the spherical Fourier Bessel domain is avoided if the frequency-radial duality (F-R) based approach is used.

The proposed methodology provides a new framework for information content for reconstruction in the spherical geometry. It is possible to optimize the reconstruction based on prior information on the bounding radius $r_{0}$. This approach proves that a smaller $r_{0}$ requires the processing of different and a fewer number of frequency samples for the same bandwidth. The Norton-Linzer solution cannot provide such an insight.

With the proposed frequency-radial duality method, we can tell which basis functions in the spatial distributions expansion is recovered for a given measurement bandwidth. This formalizes the observation that for values of lower frequency response $k_{l}$ greater than zero, large structures cannot be reconstructed but their edges are visible. These large structures contain information from the lower zero indices whose Bessel zero frequencies are lower than $k_{l}$. The Norton-Linzer method cannot be used to provide such a formal reason for this lack of reconstruction of large structures.

\section{B. Computational complexity analysis}

Firstly, we analyze the computational complexity of the time domain backprojection method presented in Ref. 2, which calculates the spatial distribution by the following operation,

$$
p\left(\boldsymbol{r}_{0}\right)=-\left.\frac{r_{0}^{2}}{2 \pi c^{2}} \int_{\Omega_{s} \in \mathbb{S}^{2}} \frac{1}{t} \frac{\partial p\left(\Omega_{s}, t\right)}{\partial t}\right|_{t=\left\|\boldsymbol{r}_{s}-\boldsymbol{r}\right\| / c} d \boldsymbol{\Omega}_{s} .
$$

This time domain method was proposed to overcome the high computational complexity of the Norton-Linzer method. $^{2}$ The operation count for this algorithm was calculated to be $\mathcal{O}\left(N_{R} N^{4}\right)$. This operation count is a result of analyzing the discretization of any backprojection method ${ }^{2,29}$ where $N_{R}$ is the number of radial samples in the reconstructed image and $N$ represents the usual order limit. Note that the number of sensors is proportional to $N^{2}$. In the literature, it is common to assume that $N_{R}=N$ and so the order is simplified to $\mathcal{O}\left(N^{5}\right)$. Other filtration or backprojection methods for the 3D reconstruction problems mentioned in Refs. 29 and 30 have a similar computational order of $\mathcal{O}\left(N^{5}\right)$. However, the time reversal methods ${ }^{31}$ have a complexity of $\mathcal{O}\left(N^{4}\right)$. In order to apply the time domain solution, the sensors must be placed in the "farfield," i.e., $|k| r_{s} \gg 1$. Both the Norton-Linzer and the proposed F-R based methods allow the sensors to be placed as close as possible to the spatial distribution resulting in a higher Signal to Noise Ratio (SNR). Further, this restriction means that the lower frequency measurements cannot be processed using the backprojection method. The backprojection algorithm is an approximate reconstruction method whereas both the Norton-Linzer and the F-R based methods are exact reconstruction methods.

A direct implementation of the Norton-Linzer method results in a computational order of $\mathcal{O}\left(N^{6}\right)$. Both the F-R based method and the Norton-Linzer method uses the spherical Fourier transform which can be optimized to speed up image reconstruction. By changing the order of summation, the inverse spherical Fourier transform for a function $f(\theta, \phi)$ defined on a unit sphere can be written as

$$
f(\theta, \phi)=\sum_{m=-N}^{N} e^{i m \phi} \sum_{n=|m|}^{N} \wp_{n m} P_{n|m|}(\cos \theta),
$$

where $P_{n|m|}$ is the normalized, associated Legendre function. The inner sum over the orders $n$ for a single value of $\theta$ includes a maximum of $N$ terms and is evaluated for $2 N$ values of $\theta$ and $2 N$ number of modes with a operation cost of $\mathcal{O}\left(N^{3}\right)$. All the sums over the orders are evaluated and then stored. Next, the sums over the modes $m$ are calculated, for a single angular position $(\theta, \phi)$, involves $2 N$ sums. Since the total number of angular positions is proportional to $N^{2}$, the computational complexity of evaluating the sums over the modes for all the angular positions is $\mathcal{O}\left(N^{3}\right)$. The overall computational order of the optimized, inverse spherical Fourier transform is $\mathcal{O}\left(N^{3}\right)+\mathcal{O}\left(N^{3}\right)=\mathcal{O}\left(N^{3}\right)$. Using a similar approach, the computational complexity of the spherical Fourier transform can be reduced to $\mathcal{O}\left(N^{3}\right)$. Moreover, a fast implementation of the inverse and the forward spherical Fourier transform was introduced in Ref. 32 with an order of $\mathcal{O}\left(N^{2} \log (N)\right)$.

We divide the Norton-Linzer method into three steps: The first step calculates the spherical Fourier transform at each of the $N_{k}$ frequency samples and has order of $\mathcal{O}\left(N^{3} N_{k}\right)$ with the optimized spherical Fourier transform. The second step calculates the radial dependent coefficient $\beta_{n m}(r)$ for each of the $N_{R}$ radial samples. For a single radial position, 
$N^{2}$ coefficients are calculated, each requiring integration over $N_{k}$ frequency samples, and so has an operation order of $\mathcal{O}\left(N^{2} N_{k}\right)$. The operation count to calculate the radial coefficients for all $N_{R}$ radial samples is $\mathcal{O}\left(N^{2} N_{k} N_{R}\right)$. The last step uses the optimized spherical Fourier transform to reconstruct the spatial distribution from the radial coefficients (37) and has an order of $\mathcal{O}\left(N^{3} N_{R}\right)$ since there are $N^{2}$ radial coefficients and $N^{2}$ angular samples for each of the $N_{r}$ radial samples. The overall order for this optimized, Norton-Linzer method is $\mathcal{O}\left(N^{3} N_{k}\right)+\mathcal{O}\left(N^{2} N_{k} N_{R}\right)+\mathcal{O}\left(N^{3} N_{R}\right)=\mathcal{O}\left(N^{3} N_{k}\right)$, assuming $N=N_{R}$.

In practice, the Norton-Linzer method needs a large number of frequency samples and so the time domain signals are zero padded before applying the Fourier transform. Whether, the Norton-Linzer method is faster than the backprojection method depends on the relationship between $N_{k}$ and $N^{2}$. If $N_{k}>N^{2}$, then the Norton-Linzer method is slower than the time domain method. Furthermore, there is no method available which gives the minimum number of frequency samples $N_{k}$ required for image reconstruction using the Norton-Linzer method.

Using the fast spherical Fourier transform, the first and the last steps now have complexity of $\mathcal{O}\left(N^{2} \log (N) N_{k}\right)$ and $\mathcal{O}\left(N^{2} \log (N) N_{R}\right)$, respectively. Therefore, applying the fast spherical Fourier transform does not change the overall complexity of the Norton-Linzer method.
Lastly, we analyze the computational complexity of our proposed F-R based approach. To utilize the advantage of the optimized spherical Fourier transform, the spherical Fourier coefficients $\wp_{n m}(k)$ are calculated for frequency samples on either side of frequency values corresponding to Bessel zeroes, and then interpolated using the linear interpolation method mentioned in (32) for each order $n$ to calculate the coefficients at frequencies corresponding to Bessel zeroes. The computational order of this operation is $\mathcal{O}\left(N_{Z k} N^{3}\right)$ where $N_{Z k}$ are the reduced number of frequency samples processed and with the fast spherical Fourier transform this reduces to $\mathcal{O}\left(N_{Z k} N^{2} \log N\right)$. Lets denote the maximum number of radial indices $\ell$ for any order by $Z_{\max }$. Calculating the spherical Fourier Bessel coefficients $\alpha_{n m \ell}$ from all the $p_{n m}\left(z_{n \ell} / r_{0}\right)$ (total number of spherical Fourier coefficients proportional to $\left.N^{2}\right)$ is of order $\mathcal{O}\left(N^{2} Z_{\max }\right)$. The synthesis step (11) can be optimized by first calculating $\beta_{n m}(r)$ from the Fourier Bessel coefficients $\alpha_{n m \ell}$ using (14). The sum is over a maximum of $Z_{\max }$ terms and is evaluated for $N_{R}$ radial samples and $N^{2}$ Fourier Bessel coefficients resulting in an operation count of $\mathcal{O}\left(N^{2} N_{R} Z_{\max }\right)$. Subsequently, an optimized or the fast spherical Fourier transform can be applied for all radial samples to give an operation count of $\mathcal{O}\left(N^{3} N_{R}\right)$ or $\mathcal{O}\left(N^{2} \log (N) N_{R}\right)$, respectively. The overall order of this method using the optimized spherical Fourier transform is $\mathcal{O}\left(N^{3} N_{Z k}\right)+\mathcal{O}\left(N^{2} Z_{\max }\right)+\mathcal{O}\left(N^{2} N_{R} Z_{\max }\right)+\mathcal{O}\left(N^{3} N_{R}\right)=\mathcal{O}\left(N^{4}\right)$, provided that $N_{Z k}=N, Z_{\max } \propto N_{R}$, and $N_{R}=N$.

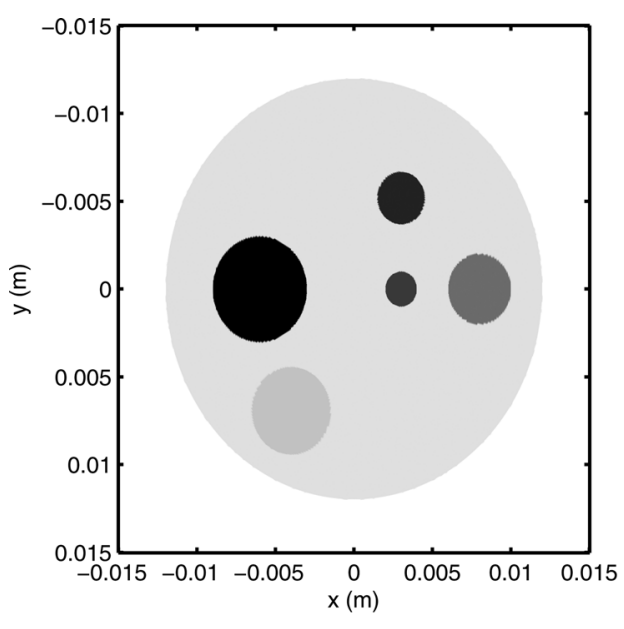

(a)

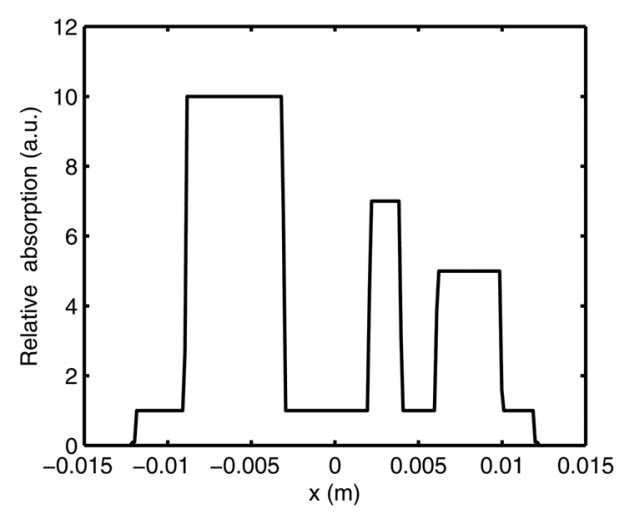

(c)

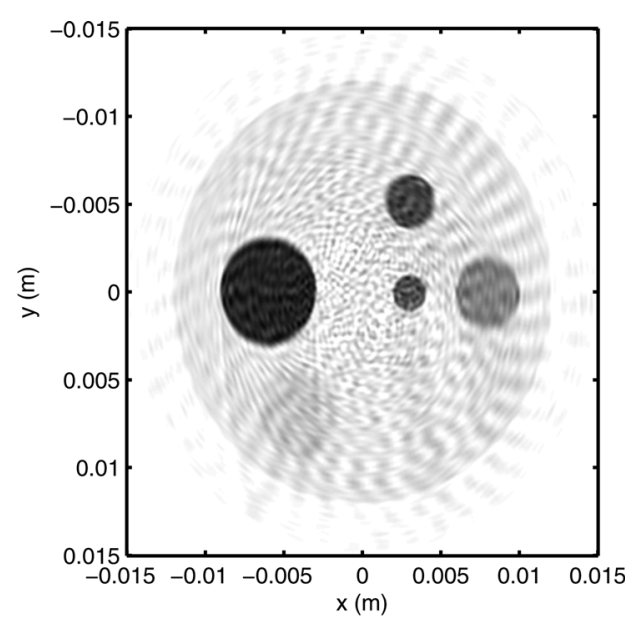

(b)

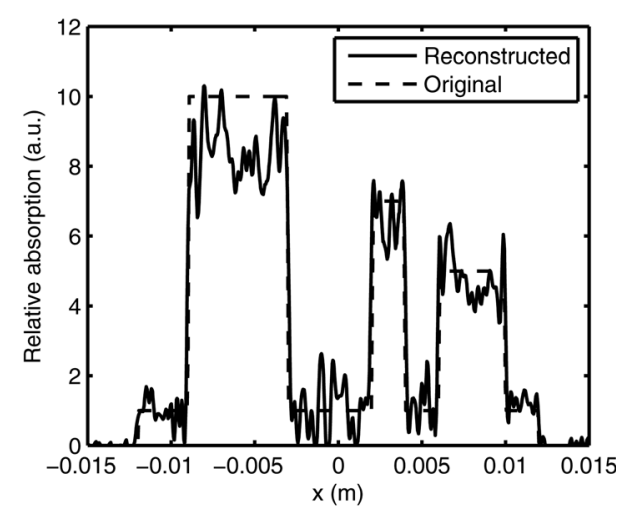

(d)
FIG. 2. Numerical phantom consisting of spherical absorbers (a) cross section through the $x-y$ plane (c) relative absorption, in arbitrary units (a.u.), through the horizontal central axis of this cross section. Reconstruction using the proposed F-R based method with frequency range from 0 to $6 \mathrm{MHz}$ and no noise (b) cross section through the $\mathrm{x}-\mathrm{y}$ plane (d) relative absorption, in arbitrary units (a.u.), through the horizontal central axis of this cross section. 
If we apply the fast spherical Fourier transform and fast transforms for the Bessel series sum (to calculate $\beta_{n m}(r)$ from the Fourier Bessel coefficients $\alpha_{n m \ell}$ ) by using fast cosine and sine transforms, ${ }^{33}$ we reduce the overall complexity of the F-R based method to $\mathcal{O}\left(N^{3} \log N\right)$. We conclude that the F-R based method is faster than both the optimized Norton-Linzer methods, the time reversal methods and the backprojection methods. The advantage of the F-R based method not apparent from the computational order analysis are the lower number of frequency samples used for each order which can speed up the reconstruction. The method to optimize the Norton-Linzer method presented in this section have not been mentioned previously in the photoacoustic literature. Depending on the number of frequency samples used, this analysis also proves that the Norton-Linzer method can be optimized to be as fast as the time reversal methods and faster than the backprojection method.

Another approach to optimizing the Norton-Linzer method was proposed in Ref. 34. This method used the Funck-Hecke formula ${ }^{35}$ to optimize the Norton-Linzer method to a similar complexity as our proposed solution. However, unlike our proposed solution, this method still requires the processing of all frequency samples for all orders in the spherical Fourier transform.

Backprojection methods can be implemented on parallel architectures such as graphical processing units (GPUs), and this is significantly faster than their single CPU implementations. ${ }^{36}$
The spherical Fourier transform can also be implemented on GPUs ${ }^{37}$ thus allowing our proposed method to be implemented on GPUs. Further, the different frequency samples can be processed by different CPUs allowing parallel implementation of the proposed method.

\section{NUMERICAL SIMULATIONS}

In this section, we describe some numerical experiments conducted to verify the validity of the proposed F-R based reconstruction method. Further, we compare the performance and the quality of the images reconstructed with the Norton-Linzer and the backprojection methods.

The numerical phantom used in the simulations is shown in Figs. 2(a) and 2(c), and consists of six spherical absorbers with centers, $r_{c}$ at the origin, $(r=3 \mathrm{~mm}$, azimuth $\phi=0$, elevation $\theta=\pi / 2),(r=8 \mathrm{~mm}, \phi=0, \theta=\pi / 2),(r=6 \mathrm{~mm}$, $\phi=\pi, \quad \theta=\pi / 2), \quad(r=8 \mathrm{~mm}, \quad \phi=2 / 3 \pi, \theta=\pi / 2)$ and $(r=6 \mathrm{~mm}, \phi=5 / 3 \pi, \theta=\pi / 2)$. These spherical absorbers are $12,1,2,3,2.5$, and $1.5 \mathrm{~mm}$ in radius, respectively. In addition, they have absorption intensities $\mu_{0}$ (in a.u.) of 1, 7, 5, 10, 2 , and 8 , respectively. In mathematical notation, the uniform spherical absorbers are normally written as $p_{0}(\boldsymbol{r})=\mu_{0} U\left(r_{a}-\left\|\boldsymbol{r}-\boldsymbol{r}_{c}\right\|\right)$, where $r_{a}$ is the radius of the sphere and the $U(\xi)$ is a unit step function which is zero when $\xi<0$ and one when $\xi \geq 0$. We assume that the speed

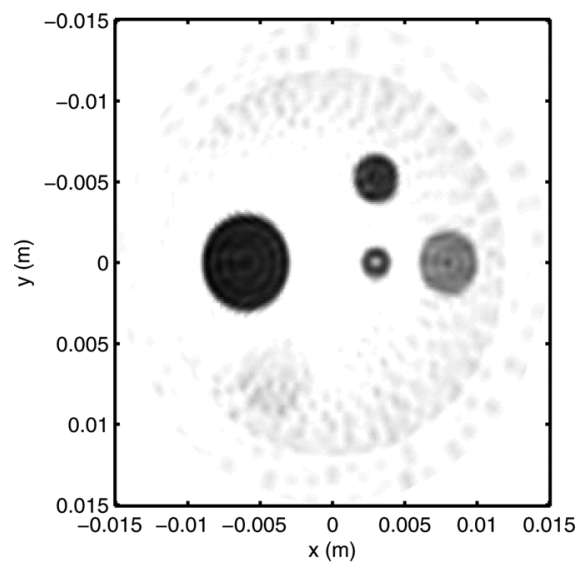

(a)

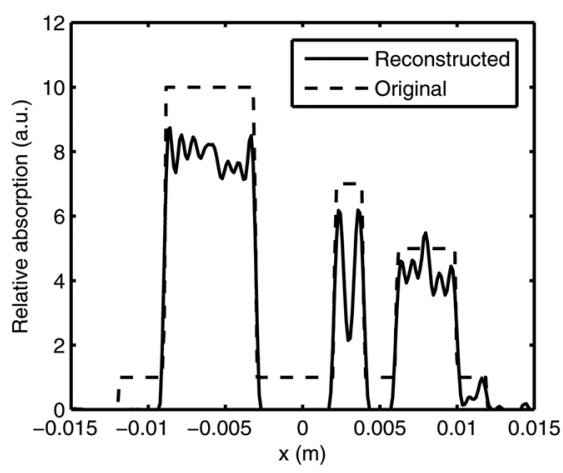

(d)

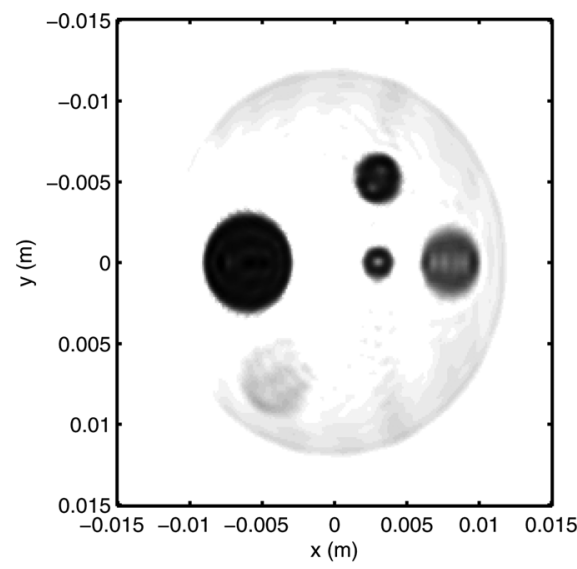

(b)

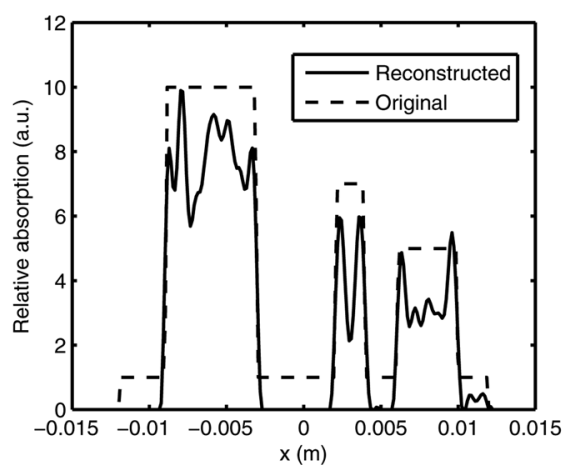

(e)

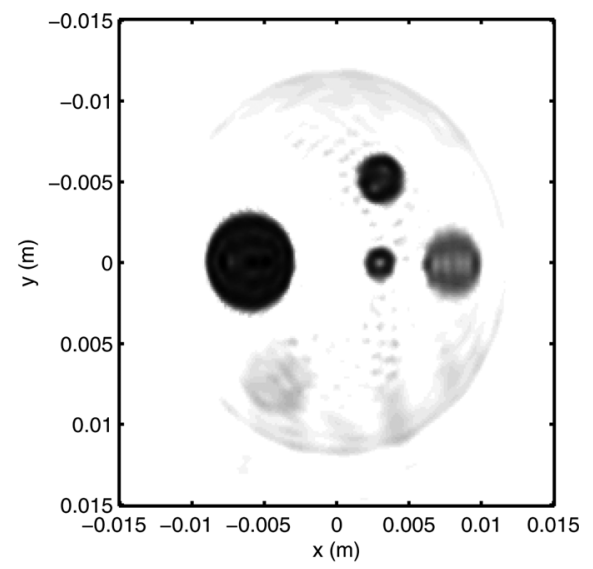

(c)

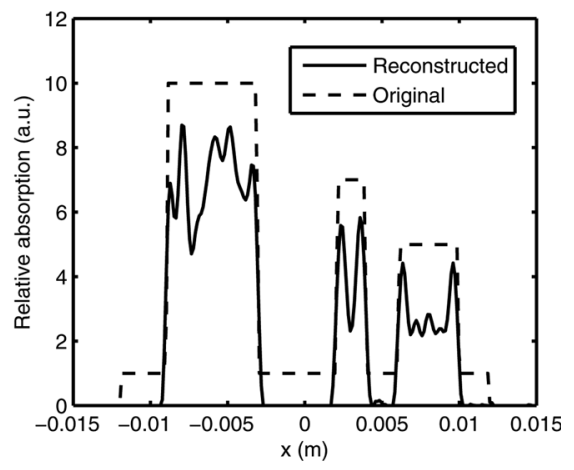

(f)

FIG. 3. Reconstruction using the three different reconstruction methods with a frequency range from $100 \mathrm{kHz}$ to $2 \mathrm{MHz}$ and $10 \mathrm{~dB}$ of noise: (a) and (d) time domain backprojection method; (b) and (e) Norton-Linzer method; and (c) and (f) F-R based method. 
of propagation is constant and is set at $c=1500 \mathrm{~m} / \mathrm{s}$, which is the typical speed of sound in biological tissue, and the optical pulse duration is short. With these two assumptions the photoacoustic signal generated by a uniform, spherical absorber is ${ }^{13,38}$

$$
p\left(\boldsymbol{r}_{s}, t\right)=\mu_{0} \Gamma U\left(r_{a}-\left|d_{\boldsymbol{r}}-c t\right|\right) \frac{d_{r}-c t}{2 d_{r}},
$$

where the distance form the center of the absorber $\boldsymbol{r}_{c}$ to the sensor position $\boldsymbol{r}_{s}$ is denoted by $d_{r}=\left\|\boldsymbol{r}_{s}-\boldsymbol{r}_{c}\right\|$ and the Grüneisen coefficient $\Gamma$ is assumed to be equal to $c^{2}$. Moreover, this formula was validated using experimental data. ${ }^{13}$

The following parameter values were chosen for the numerical experiments: the bounding radius $r_{0}$ was set to $15 \mathrm{~mm}$, the sensor radius $r_{s}$ was set to $50 \mathrm{~mm}$ and the sampling frequency was chosen to be $20 \mathrm{MHz}$. The input distribution was assumed to be order limited with a limit $N$ of 30 . Therefore, to avoid spatial aliasing, $2 N+1=62$ point sensors sample both the azimuth and the elevation with a total of 3844 sensors placed in an equiangular fashion on a sphere centered at the origin. In practice, the recorded signal can be taken by a single sensor which is rotated for several irradiation of the sample to cover the required spatial samples. Moreover, this can be an array of sensors that is rotated after the sample is radiated with a laser pulse.

The signals are recorded for $T_{s}=\left(r_{s}+r_{0}\right) / c=44 \mu \mathrm{s}$. The noise associated with photoacoustic imaging can be considered to be additive white Gaussian noise (AWGN) and is independent for the different sensor recordings. We defined the signal to noise ratio (SNR) in our experiments as

$$
S N R=10 \log _{10}\left(\frac{\int_{0}^{T_{s}}\left|p\left(\boldsymbol{r}_{\mathrm{ref}}, t\right)\right|^{2} d t}{T_{s} \sigma_{n}^{2}}\right),
$$

where $\sigma_{n}^{2}$ is the noise power and the reference sensor $\boldsymbol{r}_{\text {ref }}$ is the sensor at angular position $(\phi=0, \theta=\pi / 2)$. We used an SNR of $10 \mathrm{~dB}$.

The numerical experiments were conducted in MATLAB on a personal computer with a Intel Core 2 Duo, $2.4 \mathrm{GHz}$ processor with 2 GB of RAM. We implemented a time domain backprojection, the optimized Norton-Linzer and the optimized F-R based method. Reconstruction was done for a plane with $\theta=\pi / 2$ and 120 radial samples $N_{R}$ and also, 120 angular samples $N_{\Omega}$. Before applying the reconstruction algorithms, the time domain signal is filtered with a pass band filter equivalent to the sensor frequency response. This reduces the high frequency noise, however, better denoising algorithms can be applied if better noise suppression is required.

In the first set of simulations, the sensor frequency response is set from 0 to $6 \mathrm{MHz}$ and the reconstruction from applying the F-R based method is shown by Fig. 2(b) and Fig. 2(d). No noise was added in this numerical experiment. We can observe that each absorbing sphere is discernible and all the salient features of the numerical phantom is visible from the reconstructed image. This reconstruction shows that high frequency features can be reconstructed using the proposed method.
The second set of numerical experiments, adds $10 \mathrm{~dB}$ of noise to the sensor recordings. Further, the sensor frequency response is changed from $100 \mathrm{KHz}$ to $2 \mathrm{MHz}$, so that the restriction of $|k| r_{s} \gg 1$ is satisfied for the approximate backprojection method. The reconstructed images from using the three reconstruction methods are illustrated in Fig. 3. The Norton-Linzer method has the best quality of reconstructed image with a larger portion of the largest spherical disk being visible. The quality of the F-R based reconstruction is comparable to the Norton-Linzer method. However, the contrast in the time domain, backprojection reconstruction is lower where the absorber with absorption intensity of 2 is not visible and the reconstructed image is not as smooth as the other reconstructed images. Since the low frequency components are not used, the large spherical absorber of $12 \mathrm{~mm}$ is not fully reconstructed with only its edges being visible in all the reconstructions.

The numerical phantom is compressible in the Fourier Bessel domain, this compressibility can allow for efficient storage as well as speeding up reconstruction by using only the significant coefficients in the synthesis step of the proposed method. For a bandwidth from $100 \mathrm{KHz}$ to $2 \mathrm{MHz}$, the radial indices of 1 and 2 for order 0 and the radial index of 1 for orders 1 and 2 cannot be recovered. Therefore, the large spherical absorber cannot be fully reconstructed (Fig. 3) since the low frequency Fourier Bessel coefficients cannot be recovered. However, when these coefficients can be estimated as in the first simulation with bandwidth from 0 to $6 \mathrm{MHz}$, then this large structure is fully reconstructed [see Fig. 2(b)].

Figure 4 shows reconstruction for the partial view case using the proposed method. Similar results were obtained using the Norton-Linzer method. Reconstruction is possible only in the area covered by the sensors. The portion that is not covered cannot be reconstructed. This is also true for both the backprojection and the Norton-Linzer methods. The

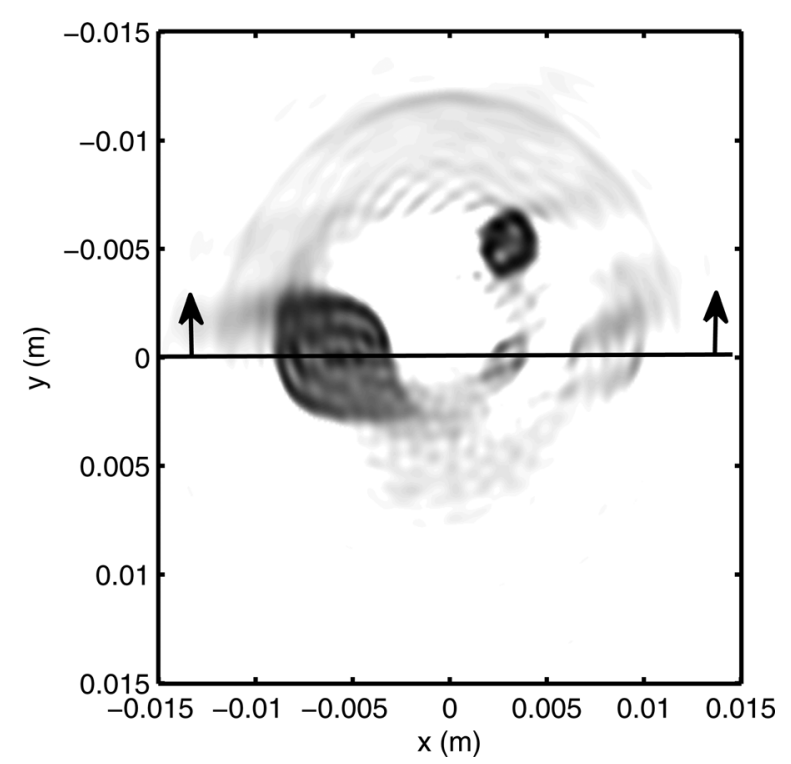

FIG. 4. Cross section of reconstruction using the proposed F-R based method with frequency range from $100 \mathrm{KHz}$ to $2 \mathrm{MHz}$ with $10 \mathrm{~dB}$ noise. A hemispherical array is used covering the upper half of the spatial distribution as shown by the arrows. 
TABLE I. Variation of run times $(\mathrm{sec})$ with frequency upper limit $(\mathrm{MHz})$ for different reconstructions methods.

\begin{tabular}{lcccccc}
\hline \hline Freq. Upper Limit & 1.0 & 1.2 & 1.4 & 1.6 & 1.8 & 2.0 \\
\hline Backprojection & 2724 & 2922 & 2879 & 2867 & 2887 & 2865 \\
Fourier Series & 125 & 153 & 180 & 217 & 267 & 319 \\
F-R Based & 25 & 28 & 32 & 37 & 44 & 53 \\
\hline \hline
\end{tabular}

proposed method was applied by setting sensor recordings that cannot be obtained in the partial view case to zero.

In the next set of simulations, we compared the speed of reconstruction for the different methods. The variation of the speed of reconstruction with the upper frequency limit is considered. The lower frequency limit was kept constant at $100 \mathrm{KHz}$. Zero padding was applied before the FFT was applied to the recorded data. The number of frequency samples that the Norton-Linzer method processes for an upper frequency limit of $2 \mathrm{MHz}$ was 305, this is about three times lower than $N^{2}=900$. In addition, the F-R based method processes a maximum of 40 frequency samples for each order, since the zero order has 40 radial indices within this bandwidth. The runtimes are depicted by Table I and Fig. 5. The F-R based method has the fastest reconstruction times, followed by the Norton-Linzer method. The rate of increase of reconstruction times with increasing frequency upper limit is slower with the F-R based method when compared to the Norton-Linzer method. The backprojection method is unaffected by the frequency upper limit. This is true provided that the samples in the reconstructed image and the number of sensors remain constant. Otherwise, the increase in frequency upper limit would require a larger number of sensors and more samples in the reconstructed image because a higher frequency bandwidth means a higher resolution of image can be reconstructed. In this experiment, we only consider the time taken to process more frequency samples between the two frequency domain methods as the bandwidth increases. From the results, the backprojection method is more than 10 times slower than the other two methods and the F-R based method is roughly about 5 times faster than the Norton-Linzer method. These results corroborate the computational order analysis performed in the previous section.

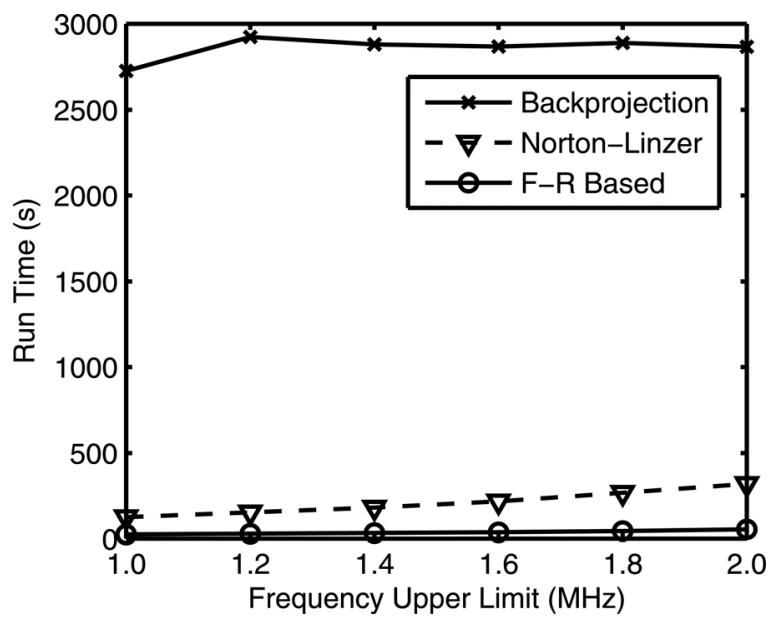

FIG. 5. Variation of run time with upper frequency limit for the different reconstruction methods.

\section{CONCLUSION}

In this work, we introduced a novel algorithm for photoacoustic image reconstruction using a spherical sensor geometry. In photoacoustics, the speed of reconstruction and the computational complexity are big factors. The proposed F-R based method, using theoretical analysis and simulations, was shown to be both less computationally complex and faster than the time domain, backprojection ${ }^{2}$ and the Norton-Linzer/Fourier series methods. The reconstructed images were of similar quality as the Norton-Linzer method and was much better than the approximate backprojection method. Moreover, unlike the approximate backprojection method, ${ }^{2}$ the spherical aperture does not have be placed in the farfield and can be placed as close as possible to the spatial distribution. The computational order analysis disapproves assumptions made in previous research that the Norton-Linzer method is slower than the backprojection method. By simple optimizations of the spherical Fourier transform, the Norton-Linzer method can be faster than the backprojection method when the number of frequency samples to be used is less than the square of the order limit.

The source distribution was expanded to a spherical Fourier Bessel domain, and the F-R based method estimates the Fourier Bessel coefficients in order to recover the initial spatial distribution. The F-R based method applies both the new concept of frequency-radial duality and modal-order filtering and is an exact reconstruction method in the continuous limit (infinite measurement bandwidth and continuous aperture) where sampling is ignored. An algorithm for discretizing this exact method for practical implementation was also presented. The concept of frequency-radial duality also proves that when the source distribution occupies a smaller region of space, the number of frequency samples that need to be used for each order is reduced. This provides a new information theoretic criteria relating volume with the number of samples that need to be processed.

\section{ACKNOWLEDGMENT}

National ICT Australia is funded through the Australian Government's Backing Australia's Ability initiative and in part through the Australian Research Council.

${ }^{1}$ S. J. Norton and M. Linzer, "Ultrasonic reflectivity imaging in three dimensions: Exact inverse scattering solution for plane, cylindrical and spherical apertures," IEEE Trans. Biomed. Eng. 28(2), 202-220 (1981).

${ }^{2} \mathrm{M}$. Xu and L. V. Wang, "Time-domain reconstruction for thermoacoustic tomography in a spherical geometry," IEEE Trans. Med. Imaging 21(7), 814-822 (2002).

${ }^{3}$ Y. Xu, D. Feng, and L. V. Wang, "Exact frequency-domain reconstruction for thermoacoustic tomography. I. Planar geometry," IEEE Trans. Med. Imaging 21(7), 823-828 (2002).

${ }^{4}$ M. Xu and L. V. Wang, "Photoacoustic imaging in biomedicine," Rev. Sci. Instrum. 77, 1-22 (2006).

${ }^{5}$ P. Liu, "The p-transform and photoacoustic image reconstruction," Phys. Med. Biol. 43(3), 667-674 (1998).

${ }^{6} \mathrm{M}$. Xu, Y. Xu, and L. V. Wang, "Time-domain reconstruction algorithms and numerical simulations for thermoacoustic tomography in various geometries," IEEE Trans. Biomed. Eng. 50(9), 1086-1099 (2003).

${ }^{7}$ K. P. Köstli and P. C. Beard, "Two-dimensional photoacoustic imaging by use of fourier-transform image reconstruction and a detector with an anisotropic response," Appl. Opt. 42(10), 1899-1908 (2003).

${ }^{8}$ S. M. A. Salehin and T. D. Abhayapala, "Frequency domain method for photoacoustic tomography with an arbitrary detection geometry," in 2010 
4th International Conference on Signal Processing and Communication Systems (ICSPCS) (IEEE, New York), pp. 1-6.

${ }^{9}$ B. T. Cox and P. C. Beard, "Photoacoustic tomography with a single detector in a reverberant cavity," J. Acoust. Soc. Am. 125(3), 1426-1436 (2009).

${ }^{10} \mathrm{G}$. B. Arfken and H. J. Weber, Mathematical Methods for Physicists (Academic, San Diego, CA, 1995), pp. 1-1182.

${ }^{11}$ R. Skomski, J. Liu, and D. Sellmyer, "Quasicoherent nucleation mode in two-phase nanomagnets," Phys. Rev. B 60(10), 7359-7365 (1999).

${ }^{12}$ B. Pons, "Ability of monocentric close-coupling expansions to describe ionization in atomic collisions," Phys. Rev. A 63, 012704 (2000).

${ }^{13}$ G. J. Diebold, T. Sun, and M. I. Khan, "Photoacoustic monopole radiation in one, two, and three dimensions," Phys. Rev. Lett. 67(24), 3384-3387 (1991).

${ }^{14}$ A. C. Tam, "Applications of photoacoustic sensing techniques," Rev. Mod. Phys. 58(2), 381-431 (1986).

${ }^{15}$ E. G. Williams, Fourier Acoustics: Sound Radiation and Nearfield Acoustical Holography (Academic, New York, 1999), pp. 1-306.

${ }^{16} \mathrm{H}$. Teutsch and W. Kellermann, "Acoustic source detection and localization based on wavefield decomposition using circular microphone arrays," J. Acoust. Soc. Am. 120(5), 2724-2736 (2006).

${ }^{17}$ S. M. A. Salehin and T. D. Abhayapala, "Localizing lung sounds: Eigen basis decomposition for localizing sources within a circular array of sensors," J. Signal Process. Syst. 64(2), 205-211 (2011).

${ }^{18}$ S. M. A. Salehin and T. D. Abhayapala, "Lung sound localization using array of acoustic sensors," in 2nd International Conference on Signal Processing and Communication Systems, 2008. ICSPCS 2008 (2008), pp. $1-5$.

${ }^{19}$ J. R. Driscoll and D. M. Healy, Jr., "Computing fourier transforms and convolutions on the 2-sphere," Adv. Appl. Math. 15(2), 202-250 (1994).

${ }^{20}$ T. D. Abhayapala, "Generalized framework for spherical microphone arrays: Spatial and frequency decomposition," in IEEE International Conference on Acoustics, Speech and Signal Processing, 2008. ICASSP 2008 (2008), pp. 5268-5271.

${ }^{21} \mathrm{Q}$. Wang, O. Ronneberger, and H. Burkhardt, "Rotational invariance based on fourier analysis in polar and spherical coordinates," IEEE Trans. Pattern Anal. Machine Intell. 31(9), 1715-1722 (2009).

${ }^{22}$ M. Liguori, A. Yadav, F. K. Hansen, E. Komatsu, S. Matarrese, and B. Wandelt, "Temperature and polarization cmb maps from primordial nongaussianities of the local type,” Phys. Rev. D 76, 105016 (2007).

${ }^{23}$ S. M. A. Salehin and T. D. Abhayapala, "Photoacoustic image reconstruction from a frequency-inavariant source localization perspective," in European Signal Processing Conference 2010, EUSIPCO2010 (2010), pp. $1627-1631$
${ }^{24}$ S. M. A. Salehin and T. D. Abhayapala, "Frequency domain, photoacoustic tomography with sparse frequency samples," in 2010 IEEE Workshop on Signal Processing Systems (SIPS), (2010), pp. 260-265.

${ }^{25}$ T. D. Abhayapala, "Generalized framework for spherical microphone arrays: Spatial and frequency decomposition," in IEEE International Conference on Acoustics, Speech and Signal Processing, 2008. ICASSP 2008 (2008), pp. 5268-5271.

${ }^{26}$ R. Hardin and N. Sloane, "Mclaren's improved snub cube and other new spherical designs in three dimensions," Discrete Comput. Geom. 15, 429-441 (1996).

${ }^{27}$ T. D. Abhayapala and D. B. Ward, "Theory and design of high order sound field microphones using spherical microphone array," in 2002 IEEE International Conference on Acoustics, Speech, and Signal Processing (ICASSP) (2002), Vol. 2, pp. 1949-1952.

${ }^{28} \mathrm{~B}$. Rafaely, "Analysis and design of spherical microphone arrays," IEEE Trans. Speech Audio Process. 13(1), 135-143 (2005).

${ }^{29}$ D. Finch, S. K. Patch, and Rakesh, "Determining a function from its mean values over a family of spheres," SIAM J. Math. Anal. 35(5), 1213-1240 (2004).

${ }^{30}$ L. Kunyansky, "Explicit inversion formulae for the spherical mean radon transform," Inverse Probl. 23, 373-383 (2007).

${ }^{31}$ Y. Hristova, P. Kuchment, and L. Nguyen, "Reconstruction and time reversal in thermoacoustic tomography in acoustically homogeneous and inhomogeneous media," Inverse Probl. 24, 055006 (2008).

${ }^{32}$ M. Tygert, "Fast algorithms for spherical harmonic expansions, II," J. Comput. Phys. 227(8), 4260-4279 (2008).

${ }^{33} \mathrm{M}$. Toyoda and T. Ozaki, "Fast spherical bessel transform via fast fourier transform and recurrence formula," Comput. Phys. Commun. 181(2), 277-282 (2010).

${ }^{34}$ L. Kunyansky, "Fast reconstruction algorithms for the thermoacoustic tomography in certain domains with cylindrical or spherical symmetries," arXiv:1102.1413, pp. 1-24 (2011).

${ }^{35}$ D. C. R. Kress and D. Colton, "Inverse acoustic and electromagnetic scattering theory," Applied Mathematical Sciences (Springer, New York, 1992), pp. 1-339.

${ }^{36}$ H. Scherl, B. Keck, M. Kowarschik, and J. Hornegger, "Fast gpu-based ct reconstruction using the common unified device architecture (cuda)," in IEEE Nuclear Science Symposium Conference Record, 2007. NSS'07 (2007), Vol. 6, pp. 4464-4466.

${ }^{37}$ A. Brunton, J. Lang, and E. Dubois, "Spherical harmonic transforms and convolutions on the gpu," J. Graphics GPU Game Tools 15(1), 13-27 (2010).

${ }^{38}$ G. J. Diebold and P. J. Westervelt, "The photoacoustic effect generated by a spherical droplet in a fluid," J. Acoust. Soc. Am. 84(6), 2245-2251 (1988). 